lack the innate antiviral response and are therefore susceptible to viral infection. However, 316Q/461G infection elicited a protective immune response against a lethal dose of the African ZIKV MR766 strain. Taken together, these observations suggest that $316 \mathrm{Q} / 461 \mathrm{G}$ has the potential to be developed as a vaccine, even for pregnant or immunocompromised individuals. Importantly, the substitutions were stably maintained in both mosquito and mammalian cells after 5 passages and the virus can be grown to high titres in $\mathrm{C} 3 / 36$ cells, further supporting its vaccine potential.

This study demonstrates that DMS can - within a few days identify not only amino acids with host-specific roles in replication but also virus strains with the potential to be developed as vaccines.

\section{Dorothy Clyde}

ORIGINAL ARTICLE Setoh, Y.X. et al. Determinants of Zika virus host tropism uncovered by deep mutational scanning. Nat. Microbiol. https://doi.org/10.1038/s41564-019-0399-4 (2019)

Last, highlighting the cumulatively large effect of the variants in the GPS, for the $1.6 \%$ of the population with the highest GPS, the increase in BMI was equivalent to large-effect pathogenic mutations in the melanocortin 4 receptor (MC4R) gene. As these MC4R variants are present in only $0.14 \%$ of the population, individuals with extremely high GPS could represent a much larger high-risk population than those harbouring pathogenic obesity-related mutations.

It will be interesting to determine what more we can learn from obesity-related predictive scores, such as whether they shed light on molecular networks underlying obesity, and the degree of clinical value they add beyond a genetically naive approach of targeting health interventions to individuals already displaying elevated BMI.

Darren J. Burgess

ORIGINAL ARTICLE Khera, A. V. et al.

Polygenic prediction of weight and obesity trajectories from birth to adulthood. Cell 177 , 587-596 (2019)

FURTHER READING Torkamani, A., Wineinger, N. E. \& Topol, E.J. The personal and clinical utility of polygenic risk scores. Nat. Rev. Genet. 19, 581-590 (2018)

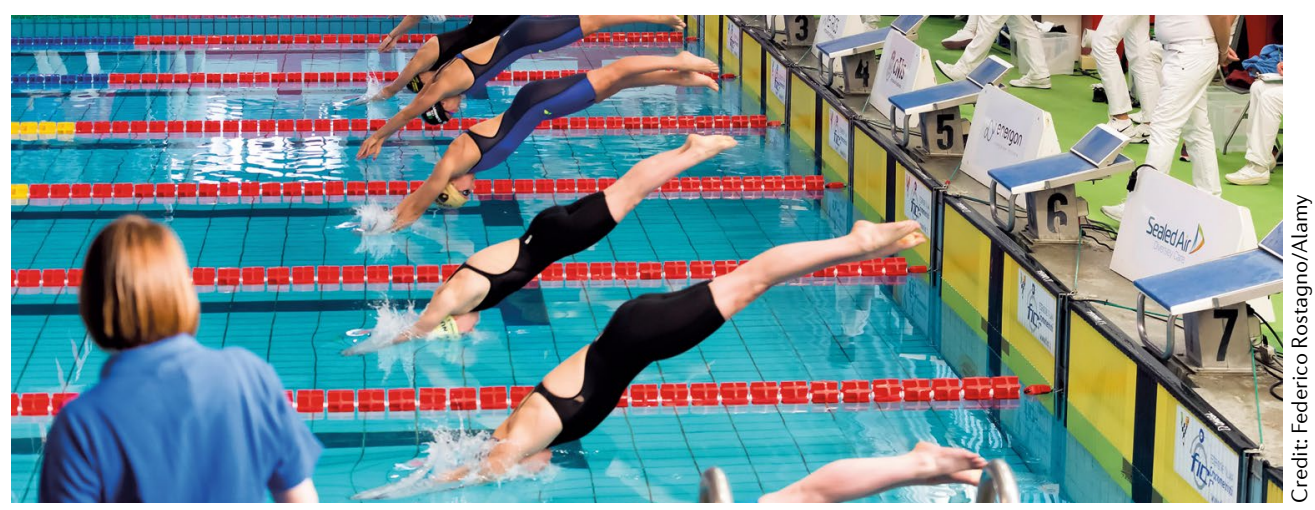

REPROGRAMMING

\title{
When the elite compete
}

A new study in Science reports the existence of a subpopulation of somatic cells from which 'elite' clones emerge that outperform other clones to drive reprogramming, the transformation of differentiated cells into pluripotent stem cells. Indeed, competitive interactions - either indirect due to competition for limited nutrients or space, or direct competition between reprogramming clones - drive clonal dynamics in a population setting in such a way that somatic cells differ in their reprogramming potential.

Previous analyses of isolated single cells undergoing reprogramming suggested that every somatic cell has the ability to reprogramme, a concept known as clonal equipotency. However, the random nature of clonal reprogramming has been hard to gel with findings from population analyses, which suggest that cells undergo deterministic steps during reprogramming. Shakiba et al. thus set out to relate cell population outcomes and single-cell reprogramming events.

By tagging mouse embryonic fibroblasts (MEFs) with a unique cellular barcode, the team were able to track the number of clones present in a cell population after induction of reprogramming and assess clonal selection. A rapid drop in the number of clones was observed, coinciding with increased cell death, and it continued to the extent that less than $10 \%$ of clones survived 30 days of reprogramming.

In contrast to the previous findings that suggested all cells have reprogramming capabilities, the analysis of surface marker profiles showed that not all clones contributed to the final cell fraction, comprising induced pluripotent stem cells. The differing reprogramming potential of cells in population analyses versus isolated conditions suggests that competitive interactions between clones have a role in shaping population dynamics.

Analysing the surviving cells after 30 days of reprogramming, the authors found that two dominant clones had emerged and made up more than $50 \%$ of the culture. To determine whether these clones exhibited selective advantages over others, the investigators analysed clone size distributions. The observed bimodal distribution of dominant versus non-dominant clones challenged the assumption of clonal equipotency, which would be expected to yield a unimodal clone size distribution.

To test whether the dynamics observed experimentally could arise owing to differences in clonal fitness, the authors looked at the outcomes of the barcoding study based on clone size. Clones that were largest after 8 days and 14 days of reprogramming exhibited greater dominance than smaller clones. However, clones that were larger at the start of reprogramming - that is, owing to random differences in barcoding — did not become dominant. Further experiments indicated that clonal dominance was present already in the MEF state.

To identify the subpopulation of 'elite' MEFs, the researchers developed a lineage tracing strategy to retrospectively identify MEFs based on Wnt1 expression, which marks MEFs derived from neural crest cells, a cell population responsible for the generation of a diverse array of cell and tissue types during vertebrate development, including skin and smooth muscle. After 3 weeks of reprogramming, MEFs derived from the neural crest formed $100 \%$ of the cell population, and these cells also had a higher probability of initiating reprogramming than non-neural crest-derived MEFs in both population analyses and in isolated clonal studies.

The implications of this study range from applications in synthetic biology to regenerative medicine. For example, a deeper understanding of the competitive ability of injected cells, that is, how these cells interact with endogenous cells and survive in a patient, may help to not only predict outcomes but also control cell competition.

Linda Koch

ORIGINAL ARTICLE Shakiba, N. et al. Cell competition during reprogramming gives rise to dominant clones. Science https://doi.org/ 10.1126/science.aan0925 (2019) 